\title{
Estaciones de aprendizaje en una clase de ELE en línea para adultos: percepciones sobre sus facilidades y dificultades
}

Recepción: 30/06/2021 | Revisión: 06/10/2021 | Aceptación: 28/01/2022 | Publicación: 01/03/2022

\author{
Melisa Soledad GIGENA \\ UNIBA \\ Viking International School - Dinamarca \\ gigenamelisa@gmail.com \\ https://orcid.org/oooo-00o3-0666-2649
}

Las didácticas específicas en tiempos de COVID-19

\author{
Azahara CUESTA GARCía \\ UNIBA \\ azaharacuestagarcia@ub.edu
}

https://orcid.org/oooo-0oo2-3530-1255

\begin{abstract}
Resumen: Dado el desafío que supone, en un escenario de aprendizaje en línea derivado de la situación pandémica, plantear un aprendizaje centrado en el alumno mediado por la tecnología, es relevante explorar cómo el uso de metodologías tales como el aprendizaje por estaciones, que involucran al alumnado de forma activa, autónoma y colaborativa, se pueden implementar en la clase de lenguas en línea. En este estudio cualitativo se analizan las percepciones sobre las facilidades y dificultades que presentó el aprendizaje por estaciones para los alumnos y profesores involucrados en dos experiencias didácticas en línea, creadas a medida en un contexto de clases privadas de ELE para adultos. Se analizaron catorce entrevistas con estudiantes, cuatro informes de observación focalizada de profesores observadores y un diario docente. La atención a diferentes estilos de aprendizaje y preferencias, la posibilidad de tomar decisiones y participar y el trabajo colaborativo son los aspectos facilitadores más destacados por los participantes, mientras que las dificultades más subrayadas giran en torno al tiempo de planificación, la gestión de los tiempos, las instrucciones y la cantidad de actividades. La experiencia muestra que es posible trasladar el aprendizaje por estaciones a la clase en línea siguiendo un enfoque centrado en el alumno.
\end{abstract}

Palabras clave: aprendizaje por estaciones; aprendizaje en línea; ELE; enseñanza de lenguas para adultos.

\section{LEARNING STATIONS IN AN ONLINE SPANISH AS A FOREIGN LANGUAGE CLASS FOR ADULTS: PERCEPTIONS ABOUT BENEFITS AND PROBLEMS}

Abstract: The pandemic has changed the educational scenario, defying teachers to use the technology to teach online lessons maintaining a learners' centered approach. Therefore, it is fundamental to explore how to implement online methodologies such as learning stations, which have the potential 
to give the learners an active, autonomous and collaborative role in the learning process. This article provides a qualitative analysis of the benefits and problems that this methodology presented for a group of students and teachers who participated in two online Spanish lessons with learning stations tailored for these groups in a private context. To achieve this, fourteen interviews with students, four guided observation reports from teacher observers and one teacher's journal were analyzed. Attention to different learning styles and preferences, opportunities to make decisions and participate and collaoration were the most highlighted aspects of this methodology, while the main problems observed were planning time, time management, instructions, and number of activities. The experience shows that it is possible to implement the methodology of learning stations online giving a central role to the student.

Keywords: learning stations; online learning; Spanish as a Foreign Language (SFL); language teaching for adults.

\section{ESTACIONS D'APRENENTATGE EN UNA CLASSE D'ELE EN LÍNIA PER A ADULTS: PERCEPCIONS SOBRE LES FACILITATS I DIFICULTATS}

Resum: Davant del repte que suposa, en un escenari d'aprenentatge en línia derivat de la situació pandèmica, plantejar un aprenentatge centrat en l'alumne mediat per la tecnologia, escau explorar com l'ús de metodologies com l'aprenentatge per estacions, que involucra l'alumnat de forma activa, autònoma i col-laborativa, es pot implementar a la classe de llengües en línia. En aquest estudi qualitatiu s'analitzen les percepcions sobre les facilitats $i$ dificultats que va presentar l'aprenentatge per estacions per als alumnes i professors involucrats en dues experiències didàctiques en línia creades ad hoc en un context de classes privades d'ELE per a adults. Es van analitzar catorze entrevistes amb estudiants, quatre informes d'observació focalitzada de professors observadors $i$ un diari docent. L'atenció a diferents estils d'aprenentatge i preferències, la possibilitat de prendre decisions i participar i el treball col-laboratiu són els aspectes facilitadors més destacats pels participants, mentre que les dificultats més destacades giren al voltant del temps de planificació, la gestió del temps, les instruccions $i$ el nombre d'activitats. L'experiència mostra que és possible traslladar l'aprenentatge per estacions a la classe en línia seguint un enfocament centrat en l'alumnat.

Paraules clau: aprenentatge per estacions; aprenentatge en línia; espanyol com a llengua estrangera (ELE); ensenyament de llengües per a adults.

\section{Introducción}

La llegada de la "enseñanza remota de emergencia" (Hodges et al., 2020) ocasionada por los efectos de la pandemia de COVID-19 ha supuesto un gran reto para la comunidad educativa: adaptar la experiencia de aprendizaje presencial a la potencialidad de las experiencias de aprendizaje en línea y/o las experiencias mixtas. Esta situación ha desafiado a muchos docentes a incorporar la tecnología para promover un proceso de enseñanza-aprendizaje centrado en el alumno, que se viese especialmente enriquecido por las posibilidades del aprendizaje en línea: la flexibilidad, la interactividad y la posibilidad del alumno para "autodirigir" su propio aprendizaje (Adedoyin y Soykan, 2020).

Particularmente en la enseñanza de lenguas extranjeras, el paso a una enseñanza en línea o mixta implica la necesidad de explorar diversas estrategias didácticas que involucren al alumno de forma activa y autónoma en la interacción generada en el aula virtual y en el apren- 
dizaje. En este contexto, resulta crucial el papel del docente como facilitador de la interacción entre los alumnos a diferentes niveles y como posibilitador de experiencias de aprendizaje y de retroalimentación personalizadas. El aprendizaje por estaciones es una de las metodologías que pueden promoverlo, y por ello, la relevancia del presente estudio radica en explorar cómo funciona el aprendizaje por estaciones en el aula en línea de lenguas extranjeras en este sentido.

En este trabajo se analizan las percepciones sobre la implementación del aprendizaje por estaciones a la clase en línea de un grupo de participantes involucrados en dos experiencias didácticas de ELE para adultos en línea (Gigena, 2019). El análisis se centra en las facilidades y las dificultades que experimentaron, en torno a la puesta en práctica de esta metodología, los alumnos y las docentes que participaron en dichas sesiones. Este estudio constituye, pues, una primera aproximación a la identificación de aquellos elementos posibilitadores y obstaculizadores del aprendizaje por estaciones en línea, que han de ser considerados en el diseño instruccional de una experiencia didáctica de estas características. Otra de las razones que justifican la pertinencia de este trabajo es que constituye una de las primeras investigaciones a partir de experiencias de aprendizaje por estaciones en contextos no presenciales de enseñanza en el ámbito de lenguas extranjeras, y en particular, de ELE. Los datos recabados en este trabajo, en un contexto de enseñanza de ELE para adultos, son además de interés porque exploran cómo funciona esta metodología con un alumnado no vinculado a la educación infantil, primaria o secundaria, para el cual suelen estar dirigidas las experiencias recogidas más frecuentemente en la bibliografía.

\section{El aprendizaje por estaciones y sus implicaciones motivacionales y metodológicas}

Bauer (2009) define el aprendizaje por estaciones como una metodología en la que el profesor organiza el contenido de una unidad o un tema en una serie de estaciones de trabajo. Estas estaciones son espacios físicos en los que se colocan información, recursos y materiales para que el alumno manipule, tanto de manera autónoma como colaborativa. El objetivo primordial de esta forma de trabajo es que el alumno trabaje en función de sus aptitudes para alcanzar los objetivos y que desarrolle sus competencias. Cabe destacar que, aunque esta metodología es mucho más frecuente en contextos de enseñanza primaria y secundaria y abarca áreas de conocimiento más allá del aprendizaje de lenguas, también puede adaptarse a la enseñanzaaprendizaje de ELE (Redmann, 2005) y, en particular, a la enseñanza de adultos. De hecho, tenemos constancia de algunas experiencias de aprendizaje por estaciones en ELE en línea (Gigena, 2019), si bien la mayoría de la bibliografía hace referencia a experiencias presenciales (Danbolt Drange, 2013; Espiñeira Caderno, 2005; Espiñeira Caderno, 2010; Riveiro, 2019; Škodová, 2020; Sommerfeldt, 2004; Teruel Fernández, 2017).

El aprendizaje por estaciones se fundamenta en los siguientes principios teóricos:

- El rol central y autónomo del alumno, en el que recae la selección de qué actividades realizar según sus posibilidades, necesidades y preferencias (Espiñeira Caderno, 2005) y la propia responsabilidad en el desempeño de estas, en las que la presencia del docente es más secundaria o focalizada en la retroalimentación. 
- La diversificación y la dinamización en el aula, generadas a partir de la transformación del espacio físico (o virtual) del aula y la organización del contenido en estaciones, que buscan atender diferentes necesidades, preferencias y estilos de aprendizaje, así como el desarrollo de distintas destrezas.

- La importancia de la motivación de los alumnos, que se genera a partir de la posibilidad de elegir (Zull, 2002), tomar decisiones y trabajar a partir de sus propias aptitudes e intereses.

- La relevancia del trabajo colaborativo, puesto que las actividades grupales o con corrección entre pares "permite aprender de los demás, conocernos mejor y valorar a otros" (Espiñeira Caderno, 2005, p. 735).

Para aplicar esta metodología, los expertos sugieren recoger datos sobre los intereses y conocimientos previos del alumno sobre el tema o unidad a tratar, definir los objetivos de aprendizaje y el contenido para alcanzarlos, crear el material para cada estación y las hojas de instrucciones, definir cuántas sesiones se van a necesitar y diseñar la evaluación según los objetivos de aprendizaje (Dreher, 2013).

Si bien la idea de circuito fue tomada del ámbito deportivo (Espiñeira Caderno, 2005; Rivera Quaresma, 2015), encontramos vínculos entre esta metodología y la pedagogía de la escuela nueva, con referentes como María Montessori, Peter Petersen, Hugo Gauding, Helen Parkhurst y Celestin Freinet. Estos pedagogos destacaron la importancia de la manipulación del material para aprender haciendo y establecieron un cambio en la concepción de los roles alumno-docente (Espiñeira Caderno, 2005; Rivera Quaresma, 2015), presente en el aprendizaje por estaciones. Otras metodologías que también fomentan la manipulación de materiales y la fragmentación del trabajo en el aula son los ambientes, centros, rincones, talleres y escritorios de aprendizaje. Estos términos aparecen a menudo utilizados de forma intercambiable con el término aprendizaje por estaciones. Sin embargo, cada una de estas metodologías tiene unas características propias que la distinguen conceptualmente del aprendizaje por estaciones y que se resumen en Gigena (2019).

Por su parte, el análisis de percepciones de estudiantes y docentes, objeto de estudio de este trabajo, se enmarca en el paradigma de los estudios del pensamiento del profesor $y$ los estudios de creencias y actitudes en la enseñanza y aprendizaje de una segunda lengua (Barcelos y Kalaja, 2011; Caro Muñoz, 2017; Ramos Méndez, 2010). Borg (2003) categoriza como cognición del profesor o teacher cognition una amplia variedad de términos que engloban la dimensión cognitiva de la enseñanza, abarcando lo que docentes y alumnos saben, creen y piensan sobre ella. Bajo este paraguas conceptual de la cognición del docente, se recogen, junto con los términos de creencias y actitudes, otros términos diversos como son las asunciones, las perspectivas o las percepciones.

Según Wesely (2012), aunque las percepciones y creencias son términos que raramente se utilizan de forma diferenciada en la literatura científica, las percepciones tienden a surgir en torno a experiencias específicas, mientras que las creencias se asumen frecuentemente como "more overarching and pervasive than perceptions" (p. 100). En este sentido, se distinguen por una parte las percepciones que abarcan la forma en la que el aprendiente se entiende a sí mismo y su propio aprendizaje y, por otra, las percepciones de la situación de aprendizaje, que incluyen la forma de experimentar y comprender los aspectos del aula, como son, por ejemplo, 
los comportamientos de los profesores (Brown, 2009). En línea con el foco de este estudio, cabe destacar también la consideración de las percepciones sobre las posibilidades de acción o affordances (Peng, 2011) como mediadoras en el desarrollo de las creencias sobre aprendizaje y enseñanza. Como afirman Barcelos y Kalaja (2011) "affordances are relational and dependent on learners' perceptions of environment" (p. 286). La percepción de posibilidades alrededor de aspectos concretos de la clase, tales como el uso de determinados métodos de enseñanza, actividades centradas en el significado o la interacción con tutores e iguales, puede propiciar la emergencia de determinadas creencias de aprendizaje (Peng, 2011).

La implementación del aprendizaje por estaciones en la clase en línea plantea una oportunidad de exploración relevante, tanto desde el punto de vista didáctico como investigador. Por una parte, las particularidades de esta metodología permiten incorporar a la clase no presencial componentes que han mostrado desempeñar un papel importante en la creación de un entorno en línea de aprendizaje motivador: las posibilidades de participación en línea, la presencia social de los alumnos y docentes implicados en la interacción en el aula y la colaboración (Hrastinski, 2009; Widjaja y Chen, 2017). La consideración de estos factores en el diseño instruccional es particularmente pertinente en contextos en los que el paso de la presencialidad a la virtualidad ha venido estimulado por la situación remota de emergencia derivada de la pandemia de COVID-19. En consecuencia, la puesta en práctica del aprendizaje por estaciones en este contexto nos permite obtener evidencias sobre cómo son experimentados estos componentes por los participantes en el aula y nos ayudan a comprender su funcionamiento desde este enfoque metodológico.

Concretamente, en el ámbito de la enseñanza de ELE encontramos diversos ejemplos de experiencias de aprendizaje en línea, particularmente en el marco de proyectos y actividades telecolaborativas (Cuesta, 2012), en el que el uso de las TIC es particularmente promovedor de la participación en línea, la presencia social y la colaboración, mediante el uso síncrono y asíncrono de diversas herramientas TIC (Concheiro et al., 2021; Espejel, 2017). Estos estudios enfatizan en la importancia que tienen, en el diseño de estas experiencias de aprendizaje, elementos como la presencia social, "as progressing through the phases (1) acquiring a social identity, (2) having purposeful communication, and (3) building relationships" (Rourke et al., 2001), y la propincuidad digital o la sensación de proximidad, participación y presencia en cualquier entorno de comunicación digital que repercute de forma positiva en la interacción (Korzernny, 1978).

\section{Preguntas de investigación}

Las preguntas de investigación a las que busca dar respuesta el presente estudio son:

- ¿Qué percepciones tienen los alumnos y docentes sobre las facilidades experimentadas en la implementación del aprendizaje por estaciones en una clase de ELE en línea?

- ¿Qué percepciones tienen los alumnos y docentes sobre las dificultades experimentadas en la implementación del aprendizaje por estaciones en una clase de ELE en línea? 


\section{Metodología}

\subsection{Tipo de investigación}

Se trata de un estudio cualitativo de carácter exploratorio con foco en conocer la percepción de todos los sujetos implicados en la experiencia en línea con el aprendizaje por estaciones, partiendo de "información sobre las percepciones, emociones, prioridades, vivencias, significados y cualidades de los participantes" (Hernández-Sampieri y Mendoza Torres, 2018, p. 9). El principal objetivo de este tipo de investigación es "que se sitúen y contextualicen los descubrimientos" (p. 10) para explorar cómo funciona la metodología del aprendizaje por estaciones, en nuestro caso.

Además, este trabajo se concibe como un estudio de caso, entendido este como "el estudio de la particularidad y de la complejidad de un caso singular, para llegar a comprender su actividad en circunstancias importantes" (Stake, 1998, p. 11). Los pilotajes de las propuestas didácticas poseen particularidades que los convierten en un objeto único de investigación. Siguiendo la clasificación de Stake (1998), enmarcamos a este como un estudio de caso intrínseco porque tiene valor en sí mismo, es decir, "no nos interesa porque con su estudio aprendamos sobre otros casos o sobre algún problema general, sino porque necesitamos aprender sobre ese caso particular" (p. 16).

\subsection{Procedimiento}

Para buscar alumnos de ELE que quieran participar voluntariamente del estudio, se realizó una publicación en grupos de Facebook de eventos de intercambio de idiomas en Argentina (Mundo Lingo y Spanglish). A quienes respondieron interesados, se les envió una prueba de nivelación y se seleccionaron aquellos participantes que tenían nivel B1 y C1. Luego, se realizó una entrevista semiestructurada por Zoom en la que fueron explicadas a los alumnos las funciones de Zoom (por ser la plataforma seleccionada en las sesiones) y la dinámica de la sesión. Además, se realizó un análisis de necesidades siguiendo el modelo de Hutchinson y Waters (1987) al que se le añadió un apartado sobre conocimientos y experiencias previas de uso con las TIC (cuestionario completo en Gigena, 2019). Esta entrevista inicial tomó aproximadamente 30 minutos y en las Tablas 1 y 2 (Anexo 1 ) se detalla la información obtenida.

\subsection{Los participantes y sus respectivos roles}

Se realizaron dos sesiones de ELE en línea de aproximadamente dos horas cada una. En la primera, participaron seis alumnos de nivel B1 y en la segunda, 12 alumnos de nivel C1. Las Tablas 1 y 2 (Anexo 1) explican en detalle el perfil de cada alumno. Brevemente las edades de los participantes oscilan entre los 22 y los 45 años. De los alumnos del grupo B1 se destaca que dos se encontraban viviendo en países hispanohablantes, dos en EE.UU., uno en Rusia y uno en Inglaterra, y que todos subrayaron su interés por los aspectos culturales de los países de habla hispana. Del grupo C1 se acentúa que uno residía en Argentina, uno en EE.UU., uno en Holanda, dos en Inglaterra y tres en Canadá, y que estaban interesados en temas de actualidad. Cabe destacar que los pilotajes se llevaron a cabo como una experiencia voluntaria con grupos que se constituyeron para el presente estudio, es decir, los alumnos no estaban previamente matriculados en cursos.

Además de los alumnos, participaron en la experiencia didáctica tres profesoras de ELE con distintos roles. Dos de las profesoras eran observadoras, una con participación completa, porque se involucró con los alumnos, y la otra con participación pasiva, porque estuvo presente 
durante la sesión, pero sin involucrarse. La observadora con participación completa era la profesora invitada, encargada de interactuar con los alumnos para vincular el tema de la clase con sus realidades (ronda 1) y guiar la comprensión del input (ronda 2) a través de una discusión guiada. Luego de cada intervención, dejaba en el chat comentarios con los errores lingüísticos más relevantes de los alumnos durante la discusión para que reflexionasen con la ayuda de la tutora. La tercera profesora era la tutora durante las sesiones, diseñadora de ambas propuestas didácticas y autora del diario de clases. Durante las sesiones, sus tareas eran asistir a los alumnos en la reflexión sobre sus errores, avisar a los grupos cuando estaba por entrar la profesora invitada, guiar la puesta en común, explicar las actividades, gestionar los tiempos, mover al profesor invitado de una sala a la otra, asesorar a los participantes tecnológicamente y estar disponible para cualquier consulta. Ambas observadoras poseen más de cinco años de experiencia en clases de ELE presenciales y virtuales con alumnos adolescentes y adultos. Por su parte, la tutora posee más de 5 años de experiencia en clases de inglés como lengua extranjera a niños, adolescentes y adultos, tanto en línea como presenciales, y dos años de experiencia en clases de español presenciales a adolescentes y adultos.

\subsection{Diseño de las experiencias didácticas en línea}

Las estaciones de trabajo fueron gestionadas a través de la función Breakout rooms de la plataforma Zoom. Antes de describir las secuencias, sintetizaremos algunos términos explicados con más detalle en la propuesta de Gigena (2019): rondas, asamblea, estaciones y circuito. Cada ronda comienza cuando los participantes son ubicados en Breakout rooms para trabajar en equipos y termina cuando todos vuelven a la sala principal, que es el momento de asamblea donde discuten las actividades realizadas y se preparan para la siguiente ronda. En cada ronda hay dos o tres estaciones con una actividad cada una. Por último, el circuito es el plan que seguir con el número de rondas y asambleas en la sesión y las estaciones en cada ronda. Por ejemplo, el circuito del grupo B1 contenía tres rondas y cuatro asambleas (una al inicio, dos entre rondas y una al final), mientras que en el circuito del grupo $\mathrm{C} 1$ había dos rondas y tres asambleas (una al inicio, una entre rondas y una al final). Además, las estaciones del grupo B1 fueron tres (aprender jugando, tenemos que hablar y videoteca/biblioteca) mientras que en grupo $\mathrm{C} 1$ eran dos porque el juego se incluyó en la asamblea. Para más detalle sobre las estaciones en cada grupo, se puede consultar Gigena (2019).

A partir de la información obtenida en el análisis de necesidades, se eligieron los temas de las sesiones, las agrupaciones de los alumnos, las estaciones y las TIC. Se decidió que las estaciones BIBLIOTECA y VIDEOTECA fuesen opcionales, puesto que algunos alumnos habían manifestado su preferencia por la lectura y otros por los videos. Para satisfacer a quienes preferían clases con más contacto con el profesor, se decidió incluir un profesor invitado. Además, se incluyeron actividades lúdicas, puesto que a todos los participantes les gustaban los juegos en las clases de ELE. En cuanto a la tecnología, se optó por herramientas conocidas por todos los participantes, como Zoom, Google Forms y Youtube, y por herramientas menos conocidas, como Kahoot y Flipgrid. Para estas últimas, se envió un documento con instrucciones de uso junto a una actividad opcional de práctica días antes de la sesión.

Se optó por el enfoque por tareas puesto que el objetivo final en ambas sesiones correspondía a una actividad de uso de la lengua representativa de una situación comunicativa fuera del aula; a saber, para el grupo B1, contar una anécdota (sobre un malentendido cultural reflexionando sobre las propias actitudes interculturales) y para el grupo $\mathrm{C} 1$, discutir un tema 
de actualidad (discusión con roles sobre tres medidas contra la expansión del coronavirus en América Latina propuestas en una reunión de la Organización Mundial de la Salud). Para que los alumnos puedan llevar a cabo la tarea final, se diseñaron una serie de tareas posibilitadoras que se agruparon en estaciones.

Para la programación de los contenidos lingüísticos de ambas secuencias se siguió el inventario para el nivel B1 del Plan Curricular del Instituto Cervantes (Instituto Cervantes, 2006) y el Marco Común Europeo de Referencia (Council of Europe, 2001). Luego, se seleccionaron las herramientas digitales siguiendo el criterio ACTIONS de Bates (2005), a saber, Accesibilidad, Costo bajo o nulo, Tecnología en función del objetivo educativo, Interactividad, políticas de la Organización o institución donde se dictan las clases, Novedad y facilidad de USO (p. 49-50). Además, la experiencia didáctica fue pilotada previamente con hispanohablantes en el mismo rango de edad que los participantes y con las profesoras observadoras, con el fin de probar el material, las actividades, las herramientas digitales y los instrumentos de recogida de datos. Gracias a esta experiencia, se incorporaron las modificaciones pertinentes a la planificación inicial. Por ejemplo, se descartó una de las tres actividades de comprensión lectora focalizada en el contenido porque llevaba mucho tiempo, se cambió un juego para asegurar la participación de todos y se descartó el uso de Edpuzzle porque no funcionaba bien al compartir pantalla. A continuación, describiremos brevemente el contenido de las dos secuencias. Para una descripción más detallada del plan de clase, consultar Gigena (2019).

\subsubsection{Secuencia de nivel B1}

Luego de la presentación inicial en la sala común, la tutora distribuyó a los alumnos en las Breakout rooms (máx. 3 por sala). En una de las estaciones de la primera ronda, cada grupo decidía entre leer un texto o ver un video y responder a preguntas de comprensión en un Google Form. En otra de las estaciones, cada integrante del grupo debía describir una comida o baile típico de su país al compañero. En una última estación, un profesor invitado ingresaba a la sala y los alumnos discutían con él sobre diferencias y malentendidos culturales. En el momento de asamblea, en la sala común, cada alumno describió su comida o baile típico mientras los compañeros escuchaban y reflexionaban sobre las propias actitudes interculturales ante lo compartido. La tutora, además de gestionar la participación del alumnado, introdujo el tema de los malentendidos culturales y proporcionó ejemplos de costumbres locales que pueden ser malinterpretadas por un turista en su país.

En la segunda ronda, en una de las estaciones jugaban a una versión de Quién Quiere Ser Millonario para repasar contenidos gramaticales y funcionales para contar y reaccionar a una anécdota. En otra de las estaciones, discutían con el profesor invitado sus respuestas sobre el video o texto para profundizar la comprensión. En una última estación, cada alumno preparaba su anécdota sobre un malentendido cultural siguiendo una infografía. En el momento de asamblea en la sala común, la tutora explicaba las actividades de la siguiente ronda. En la última ronda, en una de las estaciones, los alumnos realizaban ejercicios sobre el contenido lingüístico del texto o vídeo (expresiones para referirse a costumbres, pretérito imperfecto y conectores). En otra de las estaciones, leían una anécdota y deducían el significado de expresiones idiomáticas presentes en ella. En la última estación, cada alumno contaba su anécdota y reaccionaba a la del compañero. La clase terminó con una puesta en común sobre el juego de adivinar expresiones idiomáticas y la metodología. 


\subsubsection{Secuencia de nivel C1}

Luego de la presentación inicial en la sala común, la tutora distribuyó a los alumnos en las Breakout rooms (máx. 3 por sala) y le asignó una perspectiva a cada grupo (política, economía, salud o sociedad). En una de las estaciones de la primera ronda, cada integrante del grupo decidía entre leer un texto o ver un video sobre noticias del covid-19 en América Latina relacionadas a la perspectiva que les tocó y luego hacían una puesta en común con el compañero. En la otra estación, un profesor invitado ingresaba a la sala y reflexionaba con los alumnos sobre la pandemia en sus países. En el momento de asamblea, jugaron a Kahoot para repasar los contenidos lingüísticos necesarios en la tarea final (10 preguntas).

En la segunda ronda, en una de las estaciones, los participantes hacían una infografía con la información del input para justificar qué medida eligieron de las tres posibles (1- permitir reuniones familiares de menos de 10 personas y salidas recreativas a parques y plazas, 2-cobrar multas a quienes no cumplen la cuarentena y usar el dinero recaudado para comprar insumos médicos y para aumentarle el sueldo al personal de salud y 3 - reducir el sueldo de diputados y congresistas, y usar ese dinero para subsidiar a las personas con trabajos informales). Para ello, podían utilizar los modelos provistos en la hoja de ruta o realizar su propio diseño. En la otra estación, el profesor invitado los ayudaba a reflexionar sobre posibles modificaciones a las medidas no seleccionadas. En la sala común, terminaron el juego de Kahoot (10 preguntas más) y realizaron la tarea final: discusión con roles en simulación de reunión de la OMS. Cada pareja expuso la medida seleccionada y explicó sus razones. Luego, cada grupo propuso modificaciones a las medidas no seleccionadas y entre todos acordaron una medida.

\subsection{Instrumentos de recogida y análisis de los datos}

Las fuentes de datos recopilados y analizadas en este estudio son:

- Catorce entrevistas semiestructuradas realizadas a los alumnos, basadas en preguntas abiertas (Gigena, 2019) porque permitieron conocer sus experiencias en mayor profundidad y modificar las preguntas si era necesario (Hernández-Sampieri y Mendoza Torres, 2018). Se incluyeron varias preguntas para medir las dificultades y facilidades del aprendizaje por estaciones en la clase de ELE en línea debido a que la confiabilidad del instrumento depende de la cantidad de ítems que se incluyan para medir lo mismo (Hernández-Sampieri y Mendoza Torres, 2018). Dichas entrevistas se realizaron por Zoom días después de la sesión y fueron grabadas y transcritas utilizando una transcripción natural, es decir, omitiendo repeticiones y mejorando la coherencia y cohesión. Los alumnos tenían la libertad de responder en inglés si les resultaba más sencillo y, en promedio, estas entrevistas duraron veinte minutos.

- Cuatro informes de observación focalizada, realizados por las dos profesoras observadoras sobre las dos sesiones en línea, a partir de un listado de criterios de observación (Gigena, 2019). Se optó por las observaciones de clase dado que permiten comprender una situación analizando sus significados y actores (Hernández-Sampieri y Mendoza Torres, 2018).

- Un diario de clase semiestructurado realizado por la profesora tutora, que aborda las experiencias de la docente durante todo el proceso de diseño, 
pilotaje e implementación en el aula virtual de las dos secuencias didácticas basadas en el aprendizaje por estaciones. Dicho diario está constituido por seis entradas, de 2 a 4 páginas de extensión cada una. Se utilizó esta herramienta porque permite tener una visión holística e interna de la situación (Díaz Martínez, 1997) debido a que se analizan los comportamientos en su ambiente natural y se interpretan los significados desde el punto de vista del protagonista.

Una vez recabada la información extraída de estos tres instrumentos, se llevó a cabo un análisis temático de los datos guiado por las preguntas de investigación: se identificaron las facilidades y dificultades experimentadas en las sesiones alrededor de la metodología del aprendizaje por estaciones. Siguiendo a Hernández-Sampieri y Mendoza Torres (2018), se llevó a cabo una codificación abierta de los datos, es decir, se realizó un análisis inductivo para establecer las categorías dentro de cada tema, para, a continuación, realizar una codificación axial en la que se identificaron aquellos segmentos más representativos de cada instrumento. Esta codificación fue realizada manualmente y revisada por ambas autoras del presente trabajo (ver tablas de códigos con ejemplos en Anexo 2). Se utilizó la siguiente nomenclatura: números romanos ( $\mathrm{I}$, II, II, etc.) para las entrevistas, letras mayúsculas ( $A, B, C$, etc.) para las entradas de diario de clase y letras minúsculas del alfabeto griego $(a, \beta, \gamma$, etc.) para las observaciones. Cabe destacar que para mantener el anonimato de los alumnos se utilizaron nombres de ciudades y para identificar las observadoras se utilizaron las siglas OPC (observadora con participación completa) y OPP (observadora con participación pasiva).

Finalmente, se realiza un análisis descriptivo de los temas y categorías con segmentos que las ilustran. Este análisis parte de una triangulación de metodología y de participantes, puesto que se comparan datos obtenidos a partir de tres métodos o instrumentos con tres tipos de participantes: observadoras, alumnos y tutora.

\section{Análisis}

En este apartado se presenta el análisis de los datos obtenidos de los tres instrumentos mencionados. Se comparan sus perspectivas en cuanto a las facilidades y dificultades de la metodología del aprendizaje por estaciones en la clase de ELE en línea.

\subsection{Facilidades del aprendizaje por estaciones en una clase de ELE en línea}

Dentro de este tema se agruparon las percepciones sobre los puntos a favor que tanto los profesores como los alumnos encontraron en la implementación del aprendizaje por estaciones en las clases de ELE en línea.

4.1.1 Atención a diferentes necesidades, preferencias $y$ estilos de aprendizaje Una de las facilidades de las estaciones de aprendizaje es que la variedad de actividades y herramientas TIC permite atender a distintas necesidades, preferencias y estilos de aprendizaje. Además, los alumnos manifestaron que la variedad los ayudó a mantenerse concentrados y a practicar distintos aspectos del idioma (ejemplos I y II). Por su parte, la observadora con participación completa resaltó cuánto se involucraron los alumnos con el tema (ejemplo a). También en el diario de clases encontramos un fragmento en el que la profesora explica cómo alumnos 
con diferentes estilos de aprendizaje se sintieron más motivados en distintos momentos de la clase (ejemplo A).

(I) "The variety was very important to keep me focused because I have a short attention span" (Oxford, C1, facilidades).

(II) "Las diferentes actividades me ayudaron a practicar diferentes ámbitos del idioma, como sucede en la vida real" (Roma, B1, facilidades).

(a) "Los estudiantes conectan ideas con las de sus compañeras y se destaca la conexión emocional con el tema abordado" (OPC, C1, facilidades).

(A) "Se observó que todos los alumnos se involucraron con la propuesta participando activamente durante las sesiones, aunque su motivación variaba según los distintos momentos de la clase. Por ejemplo, New York estaba muy interesada en la discusión con la profesora invitada, pero demostraba cierto desinterés durante el último juego y Perrot estaba muy motivada durante la discusión y el juego, pero no así cuando estaban realizando la infografía" (ent. 6, facilidades).

\subsubsection{Trabajo colaborativo}

Otro aspecto a favor de esta metodología es que fomenta la interacción y colaboración entre los alumnos. Al respecto, la profesora destaca la actitud amable y cooperativa que observó en todos los grupos cuando entraba a las salas. También menciona dos ejemplos de colaboración en la sala común (ejemplo B). Además, los alumnos manifestaron que trabajar con otro compañero durante las Breakout rooms les facilitó la tarea (fragmento III) y les permitió aprender de la forma de expresarse de los otros y escuchar distintos acentos (fragmentos IV y V).

(B) "No solo en las Breakout rooms se percibía esta actitud cooperativa entre los alumnos, sino también en la sala común: 1) cuando Manchester le pidió ayuda a Londres para describir cómo se preparaba un plato típico inglés y 2) cuando Ámsterdam le pidió a Montreal que le ayude a explicar uno de los puntos de la infografía que habían hecho" (ent. 6, facilidades).

(III) "Siempre me ha gustado trabajar en parejas porque encuentro difícil trabajar en grupos grandes. En la clase, me motivaba aún más porque mi compañera hablaba perfecto" (Cambridge, C1, facilidades).

(IV) "Escuchar a mis compañeros es lo que más me gustó porque poder oír una idea que tenías en la cabeza dicha por otra persona es muy enriquecedor" (Perrot, $\mathrm{C} 1$, facilidades).

(V) "Ayudó tener parejas porque tenés otra persona para reflexionar. Además, me gustó que pude escuchar distintos acentos en mis compañeros" (Boston, C1, facilidades).

\subsubsection{Toma de decisiones por parte del alumnado}

Otro aspecto favorable en opinión del profesorado y del alumnado es que durante la sesión, la responsabilidad de tomar decisiones recae sobre el alumno (ejemplo C y VI). 
(C) "Con el grupo C1, en cambio, sentí que los alumnos tenían el control y, aunque por momentos me daba miedo que algo salga mal, decidí confiar en ellos y los resultados fueron muy satisfactorios." (ent. 6, facilidades).

(VI) "Me gustó tener la responsabilidad de tomar decisiones porque se asemeja a lo que sucede en la vida real: nadie te dice qué hacer" (Cambridge, C1, facilidades).

\subsubsection{Posibilidad de participación}

Otro aspecto posibilitador de las estaciones de aprendizaje es que se fomenta la participación de los alumnos. De hecho, tanto los alumnos como las observadoras destacaron cuánto los alumnos contribuyeron en las diferentes discusiones (fragmentos VII, $\beta$ y $y$ ).

(VII) "Me gustó porque estoy acostumbrada a la enseñanza frontal, pero en esta clase había que contribuir y no simplemente escuchar al profesor" (Roma, B1, facilidades).

$(\boldsymbol{\beta})$ "El hecho de encontrarse solos en la sala les da más oportunidades de interacción ya que el profesor no es el centro de la enseñanza" (OPP, C1, facilidades)

(y) "La profe invitada entra y cuenta cuál es la situación en Colombia. Las alumnas se ven motivadas para contar lo que sucede en sus países. La conversación es espontánea y fluida" (OPP, C1, facilidades).

\subsubsection{Variedad $y$ accesibilidad de recursos}

La observadora con participación pasiva destacó la accesibilidad de las actividades (ejemplo $(\delta))$. Además, los alumnos distinguieron el uso de un input interesante, herramientas de fácil acceso y juegos (ejemplos VIII y IX). Al respecto, en el diario de clase, la tutora hace una comparación entre ambos grupos (ejemplo D).

(ठ) "Aparentemente todos pueden acceder sin dificultades a las actividades y temas propuestos. La disponibilidad de la hoja de ruta y de la posibilidad de realizar consultas a la tutora en cualquier momento de la clase es una ventaja en caso de surgir inconvenientes" (OPP, B1, facilidades).

(VIII) "El video me pareció muy interesante porque nunca veo noticias de América Latina y también me di cuenta de que estos videos son muy accesibles y fáciles de encontrar" (Quebec, C1, facilidades).

(IX) "Kahoot me encantó y si yo pude comprenderlo cualquiera puede" (Ámsterdam, B1, facilidades).

(D) "«La principal diferencia que noté es que los alumnos del grupo C1 se involucraron más con el material (texto o video), lo cual comprobé durante sus exposiciones en la discusión, mientras que los alumnos del grupo B1 se involucraron más con los juegos, sobre todo con el último (anécdota con expresiones para adivinar)" (ent. 6, facilidades).

Cabe destacar que una ventaja del medio (en línea) para el profesor es la posibilidad de grabar las sesiones durante el momento de asamblea y dar un feedback más personalizado por email luego de la clase. De hecho, el debate del grupo C1 fue grabado y luego se les envió el video a los participantes por email junto con una devolución personalizada sobre su actuación. 
Para recapitular, los participantes de las experiencias destacaron una serie de facilidades del aprendizaje por estaciones en línea, a saber, que fomenta la atención a diferentes necesidades, preferencias y estilos de aprendizaje, la colaboración, la toma de decisiones por parte del alumno, la posibilidad de participación y una variedad y accesibilidad de recursos.

\subsection{Dificultades del aprendizaje por estaciones en una clase de ELE en línea}

En este segundo apartado se encuentran las percepciones sobre los problemas que tanto el profesorado como el alumnado encontraron en la implementación del aprendizaje por estaciones en las clases de ELE en línea.

\subsubsection{Tiempo de planificación y diseño}

La mayor dificultad para el profesor fue el tiempo de planificación y diseño del material para cada estación. Ambas sesiones duraron entre una hora y media y dos. El diseño de la sesión B1 llevó 14 horas, divididas en 5 horas de planificación, 5 horas de diseño del material, 2 horas y media de pilotaje del material en la pre-prueba piloto y una hora y media para las adaptaciones en base a la devolución de los participantes de esta instancia. El diseño de la sesión C1, en cambio, llevó seis horas: tres de planificación y tres de diseño de material. En el diario de clase, la profesora reflexiona al respecto (fragmento $\mathrm{E}$ ).

(E) "En total, la preparación de la sesión C1 me llevó 6 horas, sigue siendo un tiempo elevado, considerando lo que duró la clase, pero es viable. Creo que mientras más práctica se adquiere con la metodología, menos tiempo lleva preparar la clase" (ent. 5, dificultades).

\subsubsection{Gestión de tiempo durante la clase}

Otro inconveniente que se presentó para la tutora fue la gestión de tiempo durante la clase. La tutora reflexiona al respecto (fragmento F) y ofrece una solución: agregar un tiempo a cada ronda para poder desempeñar distintas tareas (ingresar a cada Breakout room para anunciar que pronto ingresará el profesor invitado, corregir y explicar los errores lingüísticos que el profesor invitado deja en el chat luego de cada intervención con todos los grupos, estar disponible ante posibles problemas técnicos y mover al profesor invitado de una sala a la otra).

(F) "Al igual que con la planificación, la práctica hace al maestro. En la sesión con el grupo C1 pude gestionar los tiempos sin inconvenientes porque esa era mi tercera experiencia con la metodología. De todos modos, los imprevistos en la sesión B1 me sirvieron para aprender que debería haber dejado un margen de error de al menos cinco minutos cuando planifiqué las rondas" (ent. 6, dificultades).

\subsubsection{Instrucciones}

Para cinco de los estudiantes del grupo B1, seguir las instrucciones presentó un problema porque eran muy largas (ejemplo $\mathrm{X}$ ). Al respecto, recomendaron una versión más corta o recibirlas un día antes de la clase para poder leerlas con tiempo.

(X) "Also, I think the content of the instructions that we followed was heavy. Perhaps the teacher could have one version while the students have a simplified version" (Londres, B1, dificultades) 


\subsubsection{Cantidad de actividades}

El problema de las instrucciones está vinculado con la cantidad de actividades a realizar en una sola sesión, lo cual también representó un inconveniente. Un alumno recomendó dividir las actividades en dos sesiones o sacar algunas actividades (fragmento XI)

(XI) "I wasn't always clear about what learning point was because there there many different activities. I think there should be less activities or maybe more time per activity and two lessons" (Manchester, B1, dificultades).

\subsubsection{Presencia del profesor}

Otra alumna mencionó la poca presencia del profesor como un aspecto problemático porque, en sus palabras, "no había nadie que me corrija cuando cometía errores y así no puedo aprender" (New York, B1, dificultades). Al respecto sugirió acordar con el compañero de sala cómo y cuándo corregirse.

\subsubsection{Falta de explicación lingüística de errores}

Además, una alumna y una de las observadoras (ejemplo $\varepsilon$ ) destacaron la falta de explicación lingüística sobre los errores resaltados en el feedback de la profesora invitada. Al respecto, la alumna recomendó un análisis lingüístico de los errores para entender por qué se equivocaron.

( $\varepsilon$ ) "La tutora toma los errores cometidos por los estudiantes en la conversación con la profesora invitada (ella los ha ido anotando en el chat) y reflexionan juntos sobre ellos. La corrección es solamente mostrando la opción correcta, no hay explicaciones de cómo funciona el sistema de la lengua allí" (OPP, B1, dificultades).

\subsubsection{Mismo compañero en las Breakout rooms}

El último inconveniente de las estaciones de aprendizaje para dos alumnos fue el hecho de pasar demasiado tiempo con el mismo compañero en las Breakout rooms (ejemplo XII). Sin embargo, a la mayoría le gustó que fuera el mismo compañero porque tuvieron tiempo de conocerse y entenderse para trabajar en equipo (ejemplo XIII).

(XII) "Pasamos demasiado tiempo interactuando con la misma persona y era una alumna como yo, no me corregía cuando yo cometía errores" (New York, B1, dificultades).

(XIII) "It may be interesting to try changing the groups between one round and the other, especially for those who are not comfortable with their pairs, which was absolutely not my case. In fact, I was very comfortable working with Roma because we complemented each other" (Manchester, B1, dificultades).

A modo de resumen, los problemas que las estaciones de aprendizaje presentaron para el profesor fueron el tiempo de diseño y la gestión de tiempos durante la clase. Mientras que para algunos de los alumnos en estas experiencias las instrucciones fueron largas, hubo muchas actividades para una sesión y poca presencia del profesor, faltaron explicaciones lingüísticas y estuvieron demasiado tiempo con el mismo compañero en las Breakout rooms. 


\section{Discusión}

En este estudio nos planteamos como objetivo conocer las percepciones de alumnos y profesores sobre las facilidades y dificultades de las estaciones de aprendizaje a partir de su propia experiencia en una clase piloto de ELE en línea. Los resultados revelan que los elementos de esta metodología en línea que son percibidos como posibilitadores del aprendizaje son el trabajo colaborativo, la atención a las distintas necesidades, preferencias y estilos de aprendizaje, la toma de decisiones por parte de los alumnos, la posibilidad de participación y la variedad y accesibilidad de los recursos. Como la metodología requiere el uso de distinto tipo de input y el diseño de una variedad de actividades con diferentes herramientas TIC para resolverlas, se apela a distintos estilos de aprendizaje, preferencias y necesidades. Además, al dividir a los alumnos en pares en las Breakout room, se logró que todos los alumnos participaran y se involucrasen dando su opinión y acordando con el compañero cómo resolver las distintas actividades. Para la mayoría de los alumnos, esto fue un aspecto positivo porque los preparó para lo que sucede fuera del aula y les brindó autonomía. Estas son ventajas para el profesor porque favorecen el diseño de clases dinámicas, diversas y centradas en el alumno, pero también constituyen facilidades para los alumnos porque les otorgan más responsabilidad sobre el propio proceso de enseñanza-aprendizaje.

Por el contrario, los elementos que han sido percibidos como obstaculizadores del aprendizaje por estaciones en línea son, para el profesorado, el tiempo de diseño y la gestión de tiempo durante la clase y, para el alumnado, la poca presencia del profesor, la falta de explicación lingüística de los errores, trabajar con el mismo compañero toda la sesión, las instrucciones y la cantidad de actividades. Como se mencionó en el apartado anterior, los mismos participantes hicieron sugerencias de cómo mejorar estos aspectos y algunas de esas sugerencias fueron implementadas (instrucciones).

Los datos recabados a partir de esta experiencia muestran que la implementación del aprendizaje por estaciones en el aula de ELE en línea permite que el docente pueda adquirir otras funciones, más allá de las de planificación, de gestión del aula y de control del aprendizaje que desempeña en el aula presencial (Škodová, 2020). Estas experiencias facilitan la participación de varios docentes en el aula en línea y el desempeño de otras funciones como la de promover y enriquecer la participación e interacción de los alumnos en la lengua meta y la posibilidad de proporcionar feedback inmediato, más allá del correctivo, sobre la interacción en marcha. Las posibilidades de grabación permiten además que el docente pueda enriquecer el proceso de aprendizaje proporcionando retroalimentación asincrónica a la experiencia, complementaria a la retroalimentación sincrónica e inmediata que tiene lugar en el aula. En este sentido, los datos muestran que la implementación del aprendizaje por estaciones en el aula en línea puede implicar una contribución al reto que supone la gestión de la supervisión y la evaluación en el diseño instruccional de clases en línea, dado el contexto de clases remotas de emergencia derivado de la pandemia (Adedoyin y Soykan, 2020). Las percepciones de alumnos y docentes destacan, además, la flexibilidad y la adaptación de la metodología a un aprendizaje autodirigido por el alumno, en el que puede aprender a su propio ritmo (Adedoyin y Soykan, 2020).

Las posibilidades de participación del alumnado (Hrastinski, 2009; Widjaja y Chen, 2017) en el aula virtual, tanto en calidad como en cantidad, resultan un aspecto clave para los participantes en la experiencia de aprendizaje por estaciones en línea. Las percepciones de los alumnos inciden, especialmente, en el rol que desempeña la función del docente como 
facilitador de la interacción para promover la participación del alumnado. Las percepciones de los participantes también resaltan el trabajo colaborativo que promueve la experiencia (Hrastinski, 2009; Widjaja y Chen, 2017). Los alumnos destacan particularmente cómo este trabajo colaborativo facilita su participación y la interacción en el aula y posibilita la exposición a un input más diverso, $y$, en consecuencia, útil para desarrollar competencias comunicativas.

En contraste, los datos reflejan que la presencia del docente es un aspecto no percibido por los alumnos en algunas ocasiones en el marco de esta experiencia (Widjaja y Chen, 2017; Concheiro et al., 2021). Esto se experimenta, además, como un obstáculo en el proceso de aprendizaje, particularmente asociado a determinadas funciones asignadas tradicionalmente al profesor de lengua extranjera, como la corrección inmediata de los errores lingüísticos. Por tanto, parece fundamental profundizar, por un lado, a partir de futuras experiencias, sobre cómo hacer consciente al alumnado del abanico de funciones en las que están involucrados los diversos docentes que participan en la experiencia, teniendo en consideración las creencias del alumnado en torno a las funciones fundamentales del profesor, como la gestión del aula en línea o el control de la corrección durante la interacción. En esta línea, parece también de sumo interés explorar qué tipo de estrategias docentes promueven un mayor sentimiento de propincuidad digital en el marco de la experiencia de aprendizaje por estaciones en línea (Concheiro et al., 2021).

\section{Conclusión}

A partir de las percepciones recogidas de los participantes en el análisis de los datos, consideramos una serie de implicaciones pedagógicas en la planificación de la clase en línea a partir de la metodología del aprendizaje por estaciones. Estos puntos de atención se relacionan fundamentalmente con el uso de los materiales de aprendizaje, la forma de estructurar las sesiones, las dinámicas de trabajo y el papel que desempeña el docente en el proceso de diseño e implementación de estas secuencias.

En primer lugar, se destaca la necesidad de que los materiales que se utilicen en este tipo de sesiones sean multimedia e interactivos, en formato cápsula de contenido, para que los estudiantes puedan manipularlos e interactuar con ellos de forma sencilla y autónoma a lo largo de toda la sesión. Además, es imprescindible que las herramientas tecnológicas sean elegidas en función al objetivo de aprendizaje y a la experiencia de los alumnos con la tecnología, información que se puede obtener en el análisis de necesidades.

En segundo lugar, es importante que la sesión se dinamice de forma adecuada y que se incorporen pausas frecuentes que proporcionen flexibilidad al alumno para poder transitar entre las distintas estaciones tomando sus propias decisiones en el tiempo que necesite. Además de las pausas, se sugiere incorporar un tiempo extra en la planificación de cada ronda a modo de margen de error en caso de posibles inconvenientes y realizar varias sesiones para desarrollar una unidad, combinando actividades síncronas y asíncronas.

En tercer lugar, se identifica el valor de las distintas dinámicas de trabajo para promover interacciones variadas y significativas. Se sugiere combinar actividades individuales, en parejas, en grupos pequeños y a clase abierta. En los grupos pequeños, se pueden cambiar los integrantes entre rondas o sesiones para promover la interacción con distintos compañeros.

En cuarto lugar, se distingue la relevancia del rol del docente como facilitador, tanto en el proceso, el diseño de las secuencias didácticas como en el aula virtual. En la planificación de 
las estaciones, es vital tomar como base el análisis de las necesidades y preferencias del grupo meta y de los alumnos en particular para poder considerar cómo personalizar e individualizar la experiencia. Durante la sesión, el docente ha de ser especialmente claro en las instrucciones y explicaciones y ofrecer soporte y ayuda en todo momento. Además, debe ser flexible para adaptarse a las distintas realidades que los alumnos traen al aula y a los posibles inconvenientes que puedan surgir.

Por último, se destaca la participación de la profesora invitada. Para lograr un mejor resultado con las estaciones de aprendizaje, se sugiere que la gestión no esté a cargo de un solo docente, sino que estén implicados al menos dos, o varios, con funciones claras y delimitadas. De esta manera, se puede garantizar la interacción y la retroalimentación para los alumnos.

No podemos finalizar este trabajo sin destacar algunas de sus limitaciones. Aunque se ha considerado la triangulación de los datos mediante diferentes tipos de participantes, por el carácter exploratorio del estudio, no se han podido recabar datos complementarios que permitan profundizar en la satisfacción de los alumnos respecto a la propuesta o en la relación de las percepciones de docentes y alumnos con sus propias creencias e intereses. Los datos recabados, además, se limitan a una única intervención llevada a cabo con dos grupos, de forma que resulta complejo identificar si estas percepciones de docentes y alumnos se mantendrían en un marco más continuado de implementación de esta metodología en línea. Se abren, pues, futuras líneas de investigación que pasan, por una parte, por la necesidad de realizar más estudios empíricos que vayan más allá de la descripción de las experiencias didácticas. Y, por otra parte, por la necesidad de estudios cualitativos de carácter más profundizador, basados en el análisis de entrevistas en profundidad o de diarios de alumnos y docentes durante períodos más extensos de implementación.

En el presente artículo nos hemos focalizado en el aprendizaje por estaciones en un contexto de enseñanza no formal de español como lengua extranjera en línea para adultos y hemos identificado algunas implicaciones pedagógicas para aquellos profesores de ELE que decidan probar esta metodología en sus clases. La experiencia nos ha demostrado que es posible trasladar esta metodología del contexto físico y presencial del aula, donde tradicionalmente se implementa, a la clase en línea, manteniendo el rol central del alumno en el proceso de enseñanza-aprendizaje.

\section{Referencias}

Adedoyin, O. B. y Soykan, E. (2020). Covid-19 pandemic and online learning: the challenges and opportunities. Interactive Learning Environments. https://doi.org/ghwrhe

Bates, T. (2005). Technology, e-learning and distance education (2. $\left.{ }^{\mathrm{a}} \mathrm{ed}.\right)$. Routledge.

Bauer, R. (2009). Lernen an stationen weiterentwickeln. Wege zur differenzierung und zum individuellen Lernen. Cornelsen Scriptor.

Barcelos, A. y Kalaja, P. (2011). Introduction to Beliefs about SLA revisited. System, 39(3), 281-289. https://doi.org/ckn3hp

Borg, S. (2003). Teacher cognition in language teaching: a review of research on what language teachers think, believe, know and do. Language Teaching, 36(2), 81-109. https://doi.org/cststj

Brown, A. V. (2009). Students' and teachers' perceptions of effective foreign language teaching: A comparison of ideals. Modern Language Journal, 93(1), 46-60. https://doi.org/cvt54c 
Caro Muñoz, M. (2017). Pensamiento subyacente y enseñanza de español como lengua extranjera en el sistema de enseñanza reglada de Gabón [Tesis doctoral, Universidad de Barcelona]. Dipòsit Digital de la Universitat de Barcelona. http://hdl.handle.net/10803/482201

Concheiro, P., Espejel, O. y Pujolà, J. T. (2021). Flipgrid, a video app for virtual exchange, propinquity, and language learning. Perspectiva, 39(1), 1-17. https://doi.org/hd5m

Council of Europe. (2001). Common European Framework for Languages: Learning, Teaching, Assessment. https://rm.coe.int/1680459f97

Cuesta, A. (2012). Telecolaboración y competencia intercultural en ELE: Análisis de las percepciones de estudiantes de ELE sobre una secuencia de telecolaboración entre la Universidad de Groningen (Holanda) y la Universidad de Padua (Italia). [Trabajo final de máster, Universidad de Barcelona]. Dipòsit Digital de la Universitat de Barcelona. http://hdl.handle.net/2445/129918

Díaz Martínez, J. (17-20 de septiembre de 1997). El diario como instrumento de investigación de los procesos de enseñanza-aprendizaje de lengua extranjera [Comunicación]. En Alonso, K., Gil, M. y Moreno, F. (Eds.). La enseñanza del español como lengua extranjera: del pasado al futuro (pp. 271-280). VIII Congreso Internacional de ASELE. Alcalá de Henares, España. Universidad de Alcalá. https://bit.ly/3ICKQum

Danbolt Drange, E.M. (2013). Enseñar en estaciones: Una propuesta práctica para la enseñanza de ELE. En J. Santiago Guervós y Y. González Plasencia (Eds.), La competencia pragmática y la enseñanza del español como lengua extranjera (pp. 344-354). III Congreso Internacional del Español en Castilla y León, Salamanca, España. https://bit.ly/3080MwO

Dreher, T. (2013). Lernen an Stationen-Schritt für Schritt. Sachunterricht indivuell gestalten. Westermann.

Espejel, O. (2017). Las presencias social, docente y cognitiva en la comunicación mediada por computadora en un proyecto de telecolaboración interuniversitario en el ámbito de español lengua extranjera. [Tesis doctoral. Universidad de Barcelona]. Dipòsit Digital de la Universitat de Barcelona. http://hdl.handle.net/10803/482210

Espiñeira Caderno, S. (2005). Una aplicación de la enseñanza afectiva: las estaciones de aprendizaje. En A. Álvarez, L. Barrientos, M. Braña, V. Coto, M. Cuevas, C. Hoz, I. Iglesias, P. Martínez, M. Prieto y A. Turza (Eds.). La competencia pragmática y la enseñanza del español como lengua extranjera (pp.731-740). XVI Congreso Internacional de ASELE. Oviedo, España. Universidad de Oviedo. https://bit.ly/32EryoX

Espiñeira Caderno, S. (25 de enero de 2010). Segunda parada: estaciones de aprendizaje (II). DidactiRed. https://bit.ly/3p3oszS

Gigena, M. (2019). Aprendizaje por estaciones en clases de ELE en línea a adultos. Propuestas didácticas, análisis de las percepciones de los participantes y recomendaciones prácticas [Trabajo final de máster, UNIBA]. Dipòsit Digital de la Universitat de Barcelona. http://hdl.handle.net/2445/174214

Hernández-Sampieri, R. y Mendoza Torres, C.P. (2018). Metodología de la Investigación: Las rutas cuantitativa, cualitativa y mixta. https://bit.ly/3rgBXCB

Hodges, C., Moore, S., Lockee, B., Trust, T. y Bond, A. (27 de marzo de 2020). The difference between emergency remote teaching and online learning. Educause Review. https://bit.ly/3boNzx7

Hrastinski, S. (2009). A theory of online learning as online participation. Computers y Education, 52(1), 78-82. https://doi.org/cpbcpb

Hutchison, T. y Waters, A. (1987). Chapter 6: Needs Analysis. En English for Specific Purposes: A leaning-centered approach (pp. 53-63). Cambridge University Press.

Instituto Cervantes (2006). Plan Curricular del Instituto Cervantes. https://bit.ly/3HcSAmy

Korzernny, F. (1978). A Theory of Electronic Propinquity: Mediated Communication in Organizations. Communication Research, 5(1), 3-24. https://doi.org/c7rh7b

Peng, J.E. (2011). Changes in language learning beliefs during a transition to tertiary study: the mediation of classroom affordances. System, 39(3), 314-324. https://doi.org/fpntrd 
Ramos Méndez, C. (2010). Las creencias de los alumnos: posibles implicaciones para el aula de español como lengua extranjera. En L. Miquel y N. Sans (Eds.), MarcoELE: Didáctica del Español como Lengua Extranjera, (10), 105-116. https://marcoele.com/descargas/expolingua_2006.ramos.pdf

Redmann, J. (2005). Stationenlernen: A student-centered approach to working with foreign language texts. Die Unterrichtspraxis / Teaching German, 38(2), 135-142. https://www.jstor.org/stable/20060001

Riveiro, N. (10 de octubre de 2019). Cómo incluir las estaciones de aprendizaje en el aula. ELE Internacional. https://bit.ly/33Sigel

Rivera Quaresma, S. (2015) DAS STATIONEN LERNEN: una metodología alemana y su aplicación práctica en el aula de primaria [Tesis de Grado, Universidad de Salamanca]. Repositorio Documental Gredos. http://hdl.handle.net/10366/132807

Rourke, L., Anderson, T., Garrisson, D.R. y Archer, W. (2001). Assessing social presence in asynchronous, text-based computer conferencing. Journal of Distance Education, 14(3), 51-70. https://bit.ly/3ILpPor

Škodová, J. (2020). Aprendizaje por Estaciones [Diapositiva]. PraguELE: I Jornada de Formación de profesores de ELE, Praga, República Checa. https://bit.ly/35n5oZm

Sommerfeldt, K. (2004). Un itinerario de aprendizaje: el campo semántico "la vivienda" [Resumen de la presentación]. I Encuentro práctico de profesores de ELE en Alemania, Würzburg, Alemania. https://bit.ly/3s2z1Xy

Stake, R. E. (1998). Investigación con estudio de casos. Morata. https://bit.ly/3KRWQtT

Teruel Fernández, S. (2017). Estaciones de trabajo: Buenas prácticas en movimiento [Diapositiva]. VI Congreso de la Asociación Noruega de Profesores de Español, Oslo, Noruega. https://bit.ly/3r9TI4Q

Wesely, P. M. (2012). Learner attitudes, perceptions and beliefs in language Learning. Foreign Language Annals, 45(1), 98-117. https://doi.org/gfxhf3

Widjaja, A. E. y Chen, J.V. (2017). Online Learners' Motivation in Online Learning: The Effect of Online Participation, Social Presence and Collaboration. En C. Muniarti y R. Sanjaya. Learning Technologies in Education: Issues and trends (pp. 72-93). Soegijapranata Catholic University. Zull, J. E. (2002). The art of changing the brain: enriching teaching by exploring the biology of learning . Stylus Publishing. 


\section{Anexos}

\section{Anexo 1}

Tabla 1. Descripción grupo B1

\begin{tabular}{|llllll|}
\hline & Edad & L1 & $\begin{array}{l}\text { Otras } \\
\text { lenguas }\end{array}$ & Motivación & Instrucción previa \\
\hline New York & 40 & Ruso & $\begin{array}{l}\text { Inglés (C2) y } \\
\text { alemán (B1) }\end{array}$ & $\begin{array}{l}\text { Amigos latinos en NY y } \\
\text { viajes }\end{array}$ & $\begin{array}{l}\text { 2 años en Instituto } \\
\text { Cervantes y hace 4 meses } \\
\text { clases en línea privadas }\end{array}$ \\
\hline Londres & 29 & Inglés & $\begin{array}{l}\text { Chino (A1) y } \\
\text { danés (A1) }\end{array}$ & $\begin{array}{l}\text { Empezó porque le } \\
\text { gustaba como sonaba }\end{array}$ & $\begin{array}{l}\text { 2 años en universidad } \\
\text { (hace 10 años) y hace 2 } \\
\text { años en Argentina }\end{array}$ \\
\hline Roma & 35 & Italiano & $\begin{array}{l}\text { Inglés (C2) y } \\
\text { francés (A1) }\end{array}$ & $\begin{array}{l}\text { Se mudó a Chile y está } \\
\text { buscando trabajo }\end{array}$ & $\begin{array}{l}\text { Hace 4 o 5 meses } \\
\text { autodidacta }\end{array}$ \\
\hline New Jersey & 32 & Inglés & Ruso (A1) & $\begin{array}{l}\text { Su madre lo hablaba con } \\
\text { compañeras de trabajo }\end{array}$ & $\begin{array}{l}\text { 4 o 5 años de manera } \\
\text { intermitente en distintas } \\
\text { escuelas }\end{array}$ \\
\hline Moscú & 28 & Ruso & $\begin{array}{l}\text { Inglés (C1)y } \\
\text { francés (A1) }\end{array}$ & $\begin{array}{l}\text { Empezó porque le } \\
\text { gustaba como sonaba }\end{array}$ & $\begin{array}{l}\text { 3 meses en Rusia } \\
\text { particular y 6 meses } \\
\text { mientras vivía en } \\
\text { Argentina }\end{array}$ \\
\hline Manchester & 45 & Inglés & $\begin{array}{l}\text { Francés (A2), } \\
\text { chino (B1) y } \\
\text { ruso (A1) }\end{array}$ & $\begin{array}{l}\text { Comunicarse con familia } \\
\text { de esposa argentina }\end{array}$ & $\begin{array}{l}\text { 3 años en Manchester y } \\
\text { una semana en Argentina } \\
\text { durante un viaje }\end{array}$ \\
\hline
\end{tabular}

Tabla 2. Descripción grupo C1

\begin{tabular}{|c|c|c|c|c|c|}
\hline & Edad & $\mathbf{L 1}$ & $\begin{array}{l}\text { Otras } \\
\text { lenguas }\end{array}$ & Motivación & Instrucción previa \\
\hline Montreal & 25 & Inglés & $\begin{array}{l}\text { Francés } \\
\text { (nativo), } \\
\text { italiano (C1) }\end{array}$ & Como suena & $\begin{array}{l}\text { Universidad } 1 \text { año y vivió } \\
1 \text { año en Costa Rica }\end{array}$ \\
\hline Ámsterdam & 38 & Holandés & $\begin{array}{l}\text { Inglés } \mathrm{C} 1 \\
\text { alemán } \mathrm{C} 1 \\
\text { francés } \mathrm{B} 1 \mathrm{y} \\
\text { turco B1 }\end{array}$ & $\begin{array}{l}\text { Se mudó } 1 \text { año a } \\
\text { Argentina }\end{array}$ & $\begin{array}{l}6 \text { meses en instituto y } 6 \\
\text { meses clases privadas }\end{array}$ \\
\hline Boston & 22 & Inglés & $\begin{array}{l}\text { Italiano A2, } \\
\text { portugués A1 }\end{array}$ & Se mudó a Argentina & $\begin{array}{l}\text { Hace } 15 \text { años un mes en } \\
\text { España }\end{array}$ \\
\hline Oxford & 27 & Inglés & $\begin{array}{l}\text { Portugués C, } \\
\text { chino A2, } \\
\text { francés A1 }\end{array}$ & $\begin{array}{l}\text { Hablar con la familia } \\
\text { de la madre (no se } \\
\text { anima a hablar con } \\
\text { la madre en español) }\end{array}$ & $\begin{array}{l}5 \text { o } 6 \text { años en universidad } \\
\text { (nunca estudió mucho) }\end{array}$ \\
\hline Quebec & 29 & Francés & $\begin{array}{l}\text { Inglés } \\
\text { (nativo) y } \\
\text { japonés B1 }\end{array}$ & $\begin{array}{l}\text { Le interesan los } \\
\text { idiomas y era la } \\
\text { única opción en } \\
\text { escuela }\end{array}$ & $\begin{array}{l}2 \text { años en la escuela, } 3 \\
\text { años particular para ir a } \\
\text { España ( } 1 \text { año }) \text { y casi un } \\
\text { año para ir a Arg. y Chile } \\
(1 \text { año) }\end{array}$ \\
\hline Filadelfia & 26 & Inglés & $\begin{array}{l}\text { Japonés, } \\
\text { francés, latín } \\
\text { básico }\end{array}$ & $\begin{array}{l}\text { Tuvo que aprender- } \\
\text { lo en la escuela }\end{array}$ & $\begin{array}{l}\text { Dos años en la escuela, } \\
\text { dos en la universidad y } 3 \\
\text { años viviendo en } \\
\text { Argentina (particular) }\end{array}$ \\
\hline Perrot & 26 & $\begin{array}{l}\text { Creole } \\
\text { haitiano, } \\
\text { francés e } \\
\text { inglés }\end{array}$ & $\begin{array}{l}\text { Italiano C2, } \\
\text { árabe } \mathrm{C}, \\
\text { alemán } \mathrm{C}\end{array}$ & $\begin{array}{l}\text { Le interesa aprender } \\
\text { idiomas }\end{array}$ & $\begin{array}{l}\text { Dos años en universidad } \\
\text { y luego sola }\end{array}$ \\
\hline Cambridge & 23 & Inglés & Francés C1 & $\begin{array}{l}\text { Le gustaba como } \\
\text { sonaba }\end{array}$ & $\begin{array}{l}\text { Colegio } 3 \text { años, } \\
\text { universidad } 4 \text { años, y } \\
\text { vivió en Buenos Aires } 1 \\
\text { año }\end{array}$ \\
\hline
\end{tabular}




\section{Anexo 2}

Tabla 3. Resumen de la categoría "Facilidades" con ejemplos de testimonios.

\begin{tabular}{|ll|}
\hline & \multicolumn{1}{c|}{ Facilidades } \\
\hline $\begin{array}{l}\text { Atención a } \\
\text { diferentes } \\
\text { necesidades, } \\
\text { preferencias y } \\
\text { estilos de } \\
\text { aprendizaje }\end{array}$ & $\begin{array}{l}\text { (A) «Se observó que todos los alumnos se involucraron con la propuesta } \\
\text { participando activamente durante las sesiones, aunque su motivación variaba } \\
\text { según los distintos momentos de la clase. Por ejemplo, New York estaba muy } \\
\text { desinterés durante el último juego y Perrot estaba muy motivada durante la } \\
\text { discusión y el juego, pero no así cuando estaban realizando la infografia» (ent. } 6, \\
\text { facilidades) }\end{array}$ \\
\hline $\begin{array}{l}\text { Trabajo } \\
\text { colaborativo }\end{array}$ & $\begin{array}{l}\text { (VI) «Ayudó tener parejas porque tenés otra persona para reflexionar. Además, } \\
\text { facilidades) }\end{array}$ \\
\hline $\begin{array}{l}\text { Toma de } \\
\text { decisiones por } \\
\text { parte del } \\
\text { alumnado }\end{array}$ & $\begin{array}{l}\text { (VII) «Me gustó tener la responsabilidad de tomar decisiones porque se asemeja a } \\
\text { lo que sucede en la vida real: nadie te dice qué hacer» (Cambridge, C1, facilidades). }\end{array}$ \\
\hline $\begin{array}{l}\text { Posibilidad de } \\
\text { participación }\end{array}$ & $\begin{array}{l}\text { (IX) «Me gustó porque estoy acostumbrada a la enseñanza frontal, pero en esta } \\
\text { clase había que contribuir y no simplemente escuchar al profesor» (Roma, B1, } \\
\text { facilidades). }\end{array}$ \\
\hline $\begin{array}{l}\text { Variedad y } \\
\text { accesibilidad de } \\
\text { recursos }\end{array}$ & $\begin{array}{l}\text { (XI) «El video me pareció muy interesante porque nunca veo noticias de América } \\
\text { Le encontrar» (Quebec, C1, facilidades) } \\
\text { (XII) «Kahoot me encantó y si yo pude comprenderlo cualquiera puede» } \\
\text { (Ámsterdam, B1, facilidades). }\end{array}$ \\
\hline &
\end{tabular}

Tabla 4. Resumen de la categoría "Dificultades" con ejemplos de testimonios.

\begin{tabular}{|c|c|}
\hline \multicolumn{2}{|r|}{ Dificultades } \\
\hline $\begin{array}{l}\text { Tiempo de } \\
\text { planificación y } \\
\text { diseño }\end{array}$ & $\begin{array}{l}\text { (E) «En total, la preparación de la sesión C1 me llevó } 6 \text { horas, sigue siendo un } \\
\text { tiempo elevado, considerando lo que duró la clase, pero es viable. Creo que } \\
\text { mientras más práctica se adquiere con la metodología, menos tiempo lleva } \\
\text { preparar la clase» (ent. } 5 \text {, dificultades). }\end{array}$ \\
\hline $\begin{array}{l}\text { Gestión de tiempo } \\
\text { durante la clase }\end{array}$ & $\begin{array}{l}\text { (F) «Al igual que con la planificación, la práctica hace al maestro. En la sesión con } \\
\text { el grupo } \mathrm{C} 1 \text { pude gestionar los tiempos sin inconvenientes porque esa era mi tercera } \\
\text { experiencia con la metodología. De todos modos, los imprevistos en la sesión } \mathrm{B} 1 \\
\text { me sirvieron para aprender que debería haber dejado un margen de error de al } \\
\text { menos cinco minutos cuando planifiqué las rondas» (ent. } 6 \text {, dificultades). }\end{array}$ \\
\hline Instrucciones & $\begin{array}{l}\text { (XIII) «Also, I think the content of the instructions that we followed was heavy. } \\
\text { Perhaps the teacher could have one version while the students have a simplified } \\
\text { version» (Londres, B1, dificultades) }\end{array}$ \\
\hline $\begin{array}{l}\text { Cantidad de } \\
\text { actividades }\end{array}$ & $\begin{array}{l}\text { (XIV) «I wasn't always clear about what learning point was because there were } \\
\text { many different activities. I think there should be less activities or maybe more time } \\
\text { per activity and two lessons» (Manchester, B1, dificultades) }\end{array}$ \\
\hline $\begin{array}{l}\text { Presencia del } \\
\text { profesor }\end{array}$ & $\begin{array}{l}\text { "No había nadie que me corrija cuando cometía errores y así no puedo aprender" } \\
\text { (New York, B1, dificultades) }\end{array}$ \\
\hline $\begin{array}{l}\text { Falta de } \\
\text { explicación } \\
\text { lingüística de } \\
\text { errores }\end{array}$ & $\begin{array}{l}\text { (ع) «La tutora toma los errores cometidos por los estudiantes en la conversación } \\
\text { con la profesora invitada (ella los ha ido anotando en el chat) y reflexionan juntos } \\
\text { sobre ellos. La corrección es solamente mostrando la opción correcta, no hay } \\
\text { explicaciones de cómo funciona el sistema de la lengua allì (OPP, B1, } \\
\text { dificultades) }\end{array}$ \\
\hline $\begin{array}{l}\text { Mismo compañero } \\
\text { en las breakout } \\
\text { rooms }\end{array}$ & $\begin{array}{l}\text { (XVI) «It may be interesting to try changing the groups between one round and the } \\
\text { other, especially for those who are not comfortable with their pairs, which was } \\
\text { absolutely not my case. In fact, I was very comfortable working with Roma because } \\
\text { we complemented each other» (Manchester, B1, dificultades). }\end{array}$ \\
\hline
\end{tabular}

